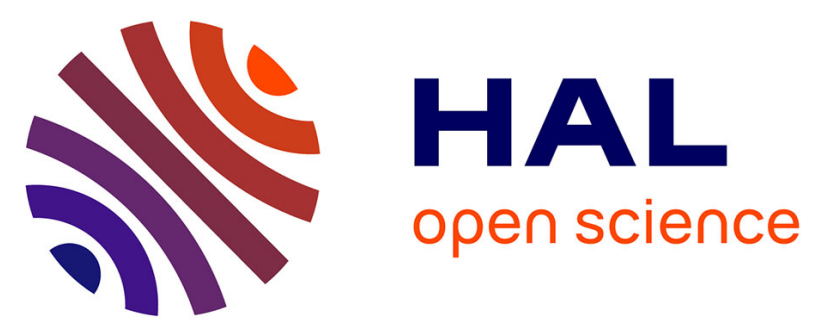

\title{
Mise en évidence de complexes associés au silicium et à l'hydrogène entrant dans le mécanisme de neutralisation des donneurs dans GaAs:Si(n) hydrogéné
} Jacques Chevallier, A. Jalil, J.C. Pesant, R. Mostefaoui, B. Pajot, P. Murawala, R. Azoulay

\section{To cite this version:}

Jacques Chevallier, A. Jalil, J.C. Pesant, R. Mostefaoui, B. Pajot, et al.. Mise en évidence de complexes associés au silicium et à l'hydrogène entrant dans le mécanisme de neutralisation des donneurs dans GaAs:Si(n) hydrogéné. Revue de Physique Appliquée, 1987, 22 (8), pp.851-857. 10.1051/rphysap:01987002208085100 . jpa-00245619

\section{HAL Id: jpa-00245619 https://hal.science/jpa-00245619}

Submitted on 1 Jan 1987

HAL is a multi-disciplinary open access archive for the deposit and dissemination of scientific research documents, whether they are published or not. The documents may come from teaching and research institutions in France or abroad, or from public or private research centers.
L'archive ouverte pluridisciplinaire HAL, est destinée au dépôt et à la diffusion de documents scientifiques de niveau recherche, publiés ou non, émanant des établissements d'enseignement et de recherche français ou étrangers, des laboratoires publics ou privés. 


\title{
Mise en évidence de complexes associés au silicium et à l'hydrogène entrant dans le mécanisme de neutralisation des donneurs dans GaAs:Si(n) hydrogéné
}

\author{
J. Chevallier $\left({ }^{+}\right)$, A. Jalil $\left({ }^{+}\right)$, J. C. Pesant $\left({ }^{+}\right)$, R. Mostefaoui $\left({ }^{+}\right)$, \\ B. Pajot $\left({ }^{\circ}\right)$, P. Murawala $\left({ }^{* \circ}\right)$ et R. Azoulay $\left({ }^{\Delta}\right)$ \\ $\left(^{+}\right)$C.N.R.S., Laboratoire de Physique des Solides, 1, place A.-Briand, 92195 Meudon-Principal Cedex, \\ France \\ $\left.{ }^{\circ}\right)$ Groupe de Physique des Solides de l'Ecole Normale Supérieure, Université Paris VII, Tour 23, 2, place \\ Jussieu, 75251 Paris Cedex 05, France \\ $\left({ }^{\Delta}\right)$ Centre National d'Etudes et Télécommunications, 196, rue de Paris, 92220 Bagneux, France
}

\begin{abstract}
Résumé. - Après exposition d'un cristal de GaAs:Si de type $\mathrm{n}$ à un plasma d'hydrogène, on constate une diminution de la concentration de porteurs libres et une diffusion d'hydrogène au voisinage de la surface du matériau. Dans les cristaux massifs, on a pu établir une bonne corrélation entre la profondeur de pénétration de l'hydrogène et la profondeur à laquelle la concentration de porteurs retrouve sa valeur de volume. La réduction de la concentration de porteurs s'accompagne d'une augmentation notable de la mobilité des électrons. Cette augmentation traduit une neutralisation des donneurs actifs par une transformation de ces donneurs en complexes neutres. Une étude détaillée par spectroscopie infrarouge de couches épitaxiées révèle une raie d'absorption étroite à $890 \mathrm{~cm}^{-1}$ dans les échantillons hydrogénés et à $637 \mathrm{~cm}^{-1}$ dans les échantillons deutérés. Ces bandes sont totalement absentes dans l'échantillon hydrogéné non dopé. L'analyse du déplacement isotopique des fréquences montre que la bande à $890 \mathrm{~cm}^{-1}$ est peut-être associée à une liaison arsenic-hydrogène où l'arsenic est supposé être en premier voisin d'un donneur silicium. Une série de recuits isochrones montre une bonne corrélation entre l'intensité de la raie à $890 \mathrm{~cm}^{-1}$ et la concentration de donneurs neutralisés dans le matériau. La neutralisation serait due à la formation de complexes $\left(\mathrm{SiAs}_{3}\right) \mathrm{As}-\mathrm{H}$, l'électron supplémentaire du silicium venant se piéger pour participer à la liaison As- $\mathrm{H}$.
\end{abstract}

\begin{abstract}
After hydrogen plasma exposure of a $n$ type GaAs:Si crystal, we observe a decrease of the free carrier concentration and a hydrogen diffusion in the near surface region of the material. In bulk crystals, a good correlation has been established between the hydrogen penetration depth and the depth where the free carrier concentration recovers its bulk value. The decrease of the carrier concentration is accompanied by a significant increase of the electron mobility. This increase reveals a neutralization of the active donors and their transformation into electrically neutral complexes. A detailed infrared spectroscopy study on plasma exposed GaAs:Si epilayers shows a very sharp absorption line at $890 \mathrm{~cm}^{-1}$ on hydrogenated samples and $637 \mathrm{~cm}^{-1}$ on deuterated samples. These bands are totally absent in hydrogenated undoped GaAs. The isotopic shift frequency analysis indicates that the $890 \mathrm{~cm}^{-1}$ line could be associated with an arsenic-hydrogen bond where arsenic is supposed to sit as a first nearest neighbour of a silicon donor. Isochronal annealing experiments show a good correlation between the $890 \mathrm{~cm}^{-1}$ absorption band intensity and the neutralized silicon donor concentration. The neutralization would be due to the formation of $\left(\mathrm{SiAs}_{3}\right) \mathrm{As}-\mathrm{H}$ complexes, the extra electron of the silicon donor being trapped in order to participate to the As-H bond.
\end{abstract}

\section{Introduction.}

On sait depuis quelques années que l'hydrogénation de semiconducteurs cristallisés tels que le silicium et l'arséniure de gallium entraîne la passivation de

$\left(^{*}\right)$ Adresse actuelle : Depart. of Physics, University of Bombay, Bombay, 400098, India. défauts. L'hydrogène atomique est capable, par exemple, de passiver les joints de grains du silicium polycristallin et les liaisons coupées situées aux interfaces $\mathrm{Si}_{-} \mathrm{SiO}_{2}[1,2]$. Plus récemment, il a été montré que l'hydrogène atomique pouvait aussi neutraliser les dopants du type accepteur dans le silicium [3,4] et dans GaAs [5] monocristallins. Dans GaAs:Si de type $\mathrm{n}$, la forte déactivation des 
donneurs dans la zone où se produit la diffusion d'hydrogène a été interprétée comme un effet de neutralisation par l'hydrogène atomique $[6,7]$. Le modèle proposé reposait sur l'hypothèse d'une interaction entre les atomes donneurs silicium et l'hydrogène atomique, interaction conduisant à l'existence de complexes impliquant le silicium et l'hydrogène.

Dans cet article, nous présentons d'abord les bases de l'effet de neutralisation. Il s'agit d'une part d'établir la correspondance entre la présence d'hydrogène et la diminution de concentration de porteurs, d'autre part de montrer pourquoi cette diminution est associée à un effet de neutralisation et non pas à un effet de compensation. Nous reportons ensuite la première observation d'une raie très fine d'absorption infrarouge dans GaAs dopé silicium et hydrogéné par plasma. Nous montrons enfin comment cette raie, associée à de l'hydrogène lié, est directement corrélée à l'effet de neutralisation.

\section{Matériaux utilisés.}

Pour les études de spectroscopie infrarouge et de neutralisation, nous avons utilisé des couches épitaxiées préparées par la technique d'épitaxie en phase vapeur à partir de composés organométalliques. Les couches ont été épitaxiées sur substrat de GaAs semi-isolant dopé chrome ou non dopé [8]. L'épaisseur des couches épitaxiées était comprise entre 3 et $4 \mu \mathrm{m}$. Les taux de dopage en silicium ont été tels que la concentration de porteurs s'étend dans la gamme $8 \times 10^{15}$ à $3 \times 10^{18} \mathrm{~cm}^{-3}$. Les études complémentaires de diffusion de deutérium ont été menées sur du matériau massif préparé par Bridgman horizontal. Le taux de dopage en silicium de ces cristaux était de $1 \times 10^{18} \mathrm{~cm}^{-3}$.

Les plasmas d'hydrogène et de deutérium ont été réalisés dans un système capacitif R.F. (13,56 MHz). L'échantillon est maintenu à $250^{\circ} \mathrm{C}$. La pression d'hydrogène dans l'enceinte à plasma est de 0,75 torr. Les temps d'exposition varient de $90 \mathrm{~min}$ à $6 \mathrm{~h}$.

\section{Diffusion d'hydrogène et neutralisation des don- neurs.}

Après exposition à un plasma de deutérium d'un monocristal massif de GaAs:Si(n) initialement homogène en concentration de porteurs $(\sim 1 \times$ $\left.10^{18} \mathrm{~cm}^{-3}\right)$, on observe une diminution sensible de cette concentration au voisinage de la surface. Cet effet peut concerner plusieurs microns suivant les conditions d'exposition. Un profil typique de concentration de porteurs est présenté sur la figure 1. Il concerne un cristal de $\mathrm{GaAs:Si}(\mathrm{n})$ exposé à $250^{\circ} \mathrm{C}$ pendant $90 \mathrm{~min}$. Ce profil a été relevé à partir d'une série de mesures capacité-tension suivies de décapages chimiques successifs. Le profil de concentration

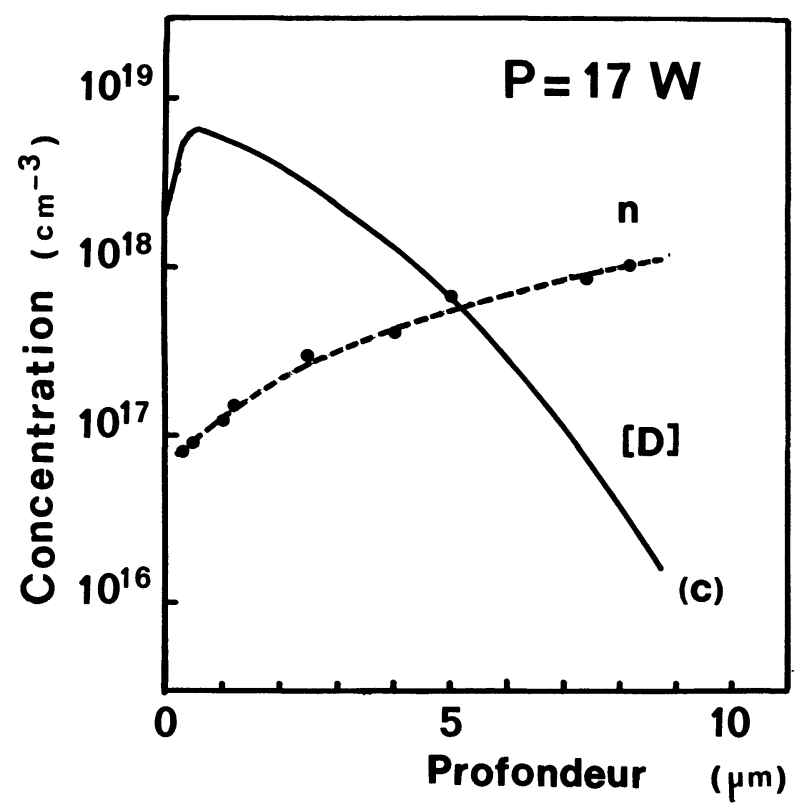

Fig. 1. - Comparaison des profils de deutérium et de concentration de porteurs libres dans un cristal massif de n-GaAs:Si exposé à un plasma de deutérium à $250^{\circ} \mathrm{C}$ pendant $90 \mathrm{~min}$. La puissance R.F. délivrée au plasma est de $17 \mathrm{~W}$

[Comparison of deuterium and free carrier concentration profiles of a n-GaAs:Si bulk sample exposed to a deuterium plasma at $250^{\circ} \mathrm{C}$ for $90 \mathrm{~min}$. The R.F. plasma power was $17 \mathrm{~W}$.]

de deutérium a été obtenu par mesures à la microsonde ionique CAMECA IMS 3f [9]. Le coefficient de diffusion du deutérium à $250^{\circ} \mathrm{C}$ est estimé à $7 \times 10^{-12} \mathrm{~cm}^{2} / \mathrm{s}[7,10]$. Le résultat le plus intéressant est la bonne corrélation, pour un cristal à dopage donné et pour des conditions d'exposition données, entre la profondeur de diffusion du deutérium et la profondeur à laquelle la concentration de porteurs retrouve sa valeur de volume [10]. On note, qu'à une profondeur fixée, la concentration de deutérium est égale ou supérieure à la variation de la concentration de porteurs. Ces résultats suggèrent que l'hydrogène joue un rôle important dans l'effet de diminution de concentration de porteurs. Cette hypothèse sera largement étayée dans la suite de ce travail.

Afin de savoir si la diminution de la concentration d'électrons était associée à un effet de neutralisation des donneurs ou à un effet de compensation, nous avons cherché à suivre l'évolution de la mobilité de porteurs après diffusion d'hydrogène dans le matériau. Dans ce but, nous avons caractérisé une série de couches épitaxiées de GaAs:Si par mesures d'effet Hall et de conductivité avant et après exposition à un plasma d'hydrogène d'une durée de $2 \times 6 \mathrm{~h}$. Après la première exposition de $6 \mathrm{~h}$, la concentration de porteurs est, comme on s'y attend, sensiblement plus faible que la concentration de porteurs initiale. Dans le cas d'un cristal de dopage 
initial $1,2 \times 10^{18} \mathrm{~cm}^{-3}$, la concentration après une telle exposition est de $5,2 \times 10^{16} \mathrm{~cm}^{-3}$ et la mobilité des électrons de $2600 \mathrm{~cm}^{2} / v$.s. La concentration de porteurs et la mobilité électronique ne varient pas entre la première et la deuxième exposition. Ceci conduit à penser qu'une exposition de $6 \mathrm{~h}$ est suffisante pour atteindre une saturation de l'effet de neutralisation. La figure 2 présente une courbe classique mobilité-température pour une couche de GaAs:Si dont la concentration de porteurs avant exposition est de $1,2 \times 10^{18} \mathrm{~cm}^{-3}$. La mobilité atteint un maximum $\mu_{\max }$ de $2700 \mathrm{~cm}^{2} / \mathrm{v}$.s. vers $150 \mathrm{~K}$.

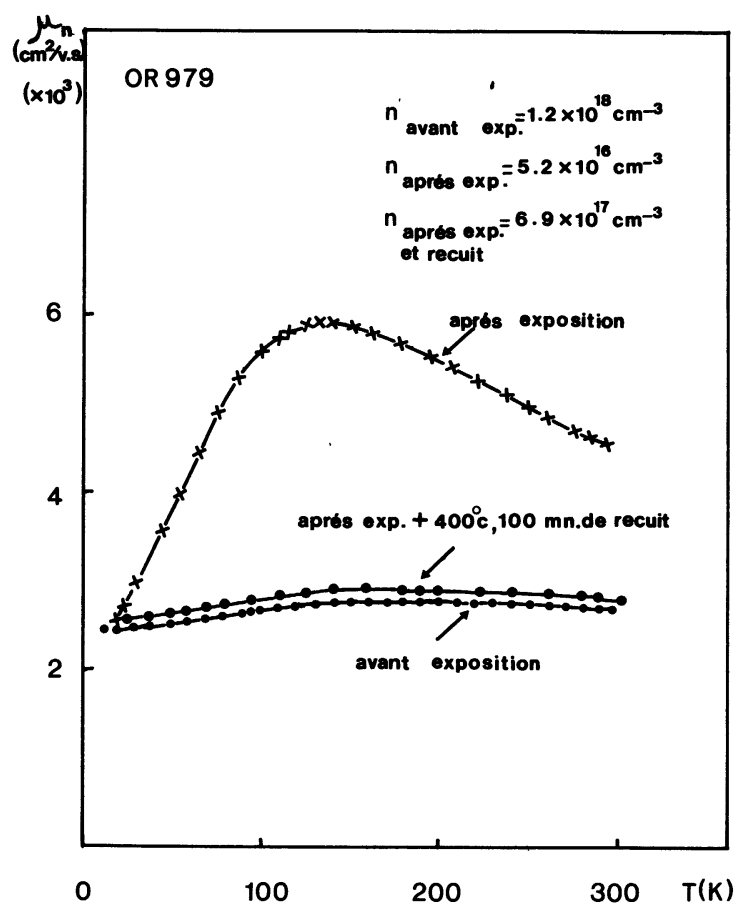

Fig. 2. - Evolution de la mobilité électronique avec la température pour une couche épitaxiée de GaAs:Si avant hydrogénation $(\bullet)$, après une exposition de $2 \times 6 \mathrm{~h}(\times)$ et après exposition suivie d'un recuit thermique à $400^{\circ} \mathrm{C}$ pendant $100 \mathrm{~min}($.$) .$

[Mobility vs. temperature curves for a GaAs:Si epilayer before any hydrogenation $(\bullet)$, after a $2 \times 6 \mathrm{~h}$ exposure $(x)$ and after exposure followed by a $400{ }^{\circ} \mathrm{C}, 100 \mathrm{~min}$ annealing (.).]

Après exposition, la mobilité est de $5900 \mathrm{~cm}^{2} / \mathrm{v} . \mathrm{s}$. vers $130 \mathrm{~K}$. D'autres couches épitaxiées, moins dopées silicium, ont été exposées à un plasma d'hydrogène identique. Les valeurs de $\mu_{\max }$ avant et après hydrogénation de chaque couche épitaxiée sont reportées sur la figure 3 en fonction de la concentration de porteurs correspondante. On note un bon accord entre les valeurs de $\mu_{\max }$ mesurées après hydrogénation et la courbe $\mu_{\max }(n)$ déduite des mesures avant hydrogénation. En d'autres termes, la mobilité des électrons dans les matériaux

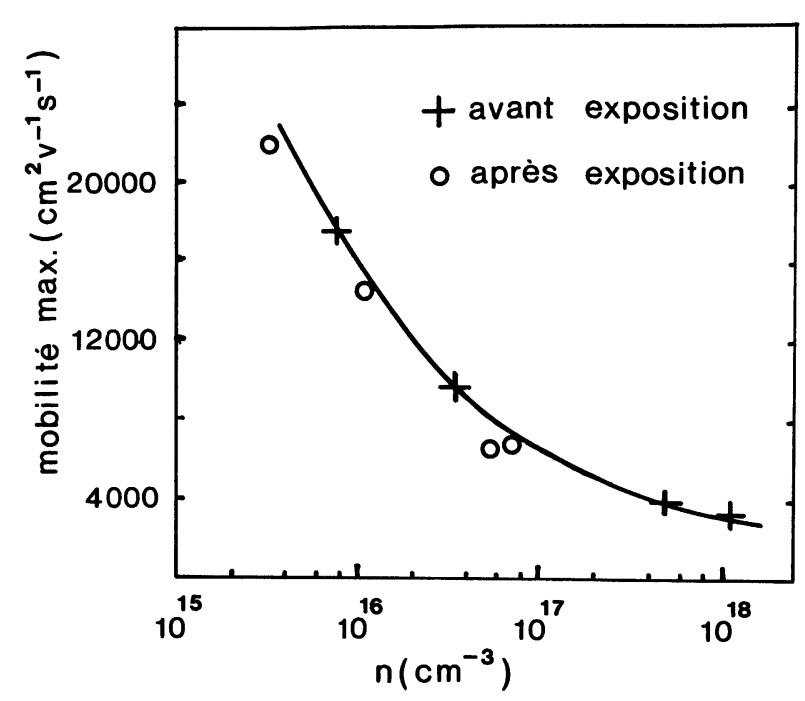

Fig. 3. - Evolution de la valeur de la mobilité maximum avec la concentration de porteurs libres pour 4 couchés épitaxiées de GaAs:Si de différents dopages, avant (+) et après (O) hydrogénation.

[Evolution of the maximum mobility value versus the free carrier concentration for $4 \mathrm{GaAs:Si}$ epilayers with different doping levels, before $(+)$ and after $(O)$ hydrogenation.]

hydrogénés est sensiblement la même, pour $3 \times 10^{15} \mathrm{~cm}^{-3}<n<10^{18} \mathrm{~cm}^{-3}$, que celle mesurée dans les matériaux non hydrogénés de concentration de porteurs équivalente. Une analyse détaillée de ces résultats [11] montre que l'augmentation de mobilité après exposition s'explique par une diminution de la concentration de donneurs ionisés. La diminution de la concentration d'électrons et la forte augmentation de mobilité traduisent qu'une fraction importante des donneurs silicium actifs électriquement s'est transformée en espèces neutres, les électrons libres venant se piéger sur les complexes formés. Notons que cet effet de neutralisation peut trouver son application en microélectronique ou en optoélectronique : un matériau à l'origine $\mathrm{n}^{+}$peut devenir n par simple exposition à un plasma d'hydrogène, le matériau possédant alors la mobilité correspondant à son nouveau dopage.

Nous avons remarqué aussi que la neutralisation des donneurs silicium était, dans des conditions de plasma données, légèrement plus profonde pour les échantillons hydrogénés que pour les échantillons deutérés. Le rapport des profondeurs est typiquement de 1,4. L'efficacité de neutralisation reste pratiquement la même. Cette variation de la profondeur de neutralisation s'explique bien par la masse plus élevée du deutérium et donc son coefficient de diffusion $\sqrt{2}$ fois plus faible que celui de l'hydrogène. Dans leurs études sur l'implantation d'hydrogène ou de deutérium dans GaAs, Steeples et al. [12] ont trouvé, pour une dose donnée, des effets de compensation très différents pour le deutérium et 
pour l'hydrogène. Ils ont attribué ce résultat à l'influence des mécanismes de recombinaison non radiative de paires électron-trou, créés pendant l'implantation, sur la concentration de défauts structuraux du cristal. Nos résultats expérimentaux confirment ainsi l'origine différente des mécanismes de neutralisation (plasma) et de compensation (implantation).

\section{Spectroscopie d'absorption infrarouge.}

Dans notre modèle initial [6], nous avions proposé que la formation de complexes neutres impliquant le silicium et l'hydrogène était à l'origine de l'effet de neutralisation. Aucune preuve directe n'avait cependant été apportée jusqu'à ce jour à la validité de ce modèle. Aussi avons-nous entrepris une étude de spectroscopie d'absorption infrarouge de façon à détecter les modes vibrationnels de ces complexes. Dans ce but, nous avons exposé à un plasma d'hydrogène ou de deutérium une série de couches épitaxiées de 3 à $4 \mu \mathrm{m}$ d'épaisseur dont le niveau de dopage en silicium était situé dans la gamme 1 à $3 \times 10^{18} \mathrm{~cm}^{-3}$. Les matériaux hydrogénés ont ensuite été analysés d'une part à $300 \mathrm{~K}$ sur un spectromètre Perkin-Elmer 683 dans la configuration de réflexion interne multiple (R.I.M.), d'autre part en simple transmission sur un spectromètre à transformée de Fourier (S.T.F.) du type BOMEM DA3.01 équipé d'un cryostat à température variable permettant de travailler entre 6 et $300 \mathrm{~K}$.

La figure 4 présente le spectre d'absorption d'une couche épitaxiée de GaAs de type $\mathrm{n}$, hydrogéné pendant $90 \mathrm{~min}$ à $250^{\circ} \mathrm{C}$. Ce spectre a été obtenu par S.T.F. On peut noter la finesse de la raie à basse température. Le relevé des spectres à $7 \mathrm{~K}$ et $77 \mathrm{~K}$ dans les conditions de haute résolution indique une largeur à mi-hauteur de $0,08 \mathrm{~cm}^{-1}$. Cette largeur est de $1,5 \mathrm{~cm}^{-1}$ à $300 \mathrm{~K}$. La raie est située à $896,8 \mathrm{~cm}^{-1}$ à $7 \mathrm{~K}$ et à $889,9 \mathrm{~cm}^{-1}$ à $300 \mathrm{~K}$. La figure $5 \mathrm{~b}$ montre la courbe de transmission d'une couche épitaxiée, fortement dopée silicium et hydrogénée, dans la configuration R.I.M. La largeur de raie est limitée dans ce cas par la résolution du spectromètre PerkinElmer $\left(\sim 2 \mathrm{~cm}^{-1}\right)$. Pour l'étude dans cette configuration, les cristaux de $\mathrm{GaAs}$ ont des dimensions typiques de $15 \times 7 \times 1,5 \mathrm{~mm}$. Ils sont taillés en biseau à $45^{\circ}$ aux deux extrémités. Cette configuration permet d'augmenter par un facteur $\sim 20$ le chemin optique dans la couche épitaxiée.

Afin de préciser la nature chimique du ou des atomes impliqués dans la vibration, nous avons exposé les mêmes matériaux à un plasma de deutérium. Après deutération, le spectre de transmission observé est celui de la figure 5c. Une raie unique apparaît à $637 \mathrm{~cm}^{-1}$ à $300 \mathrm{~K}$. Les mesures de S.T.F. indiquent une valeur précise de $636,7 \mathrm{~cm}^{-1}$. Le rapport isotopique $\nu(\mathrm{H}) / \nu(\mathrm{D})$ est de 1,398 , c'est-à-

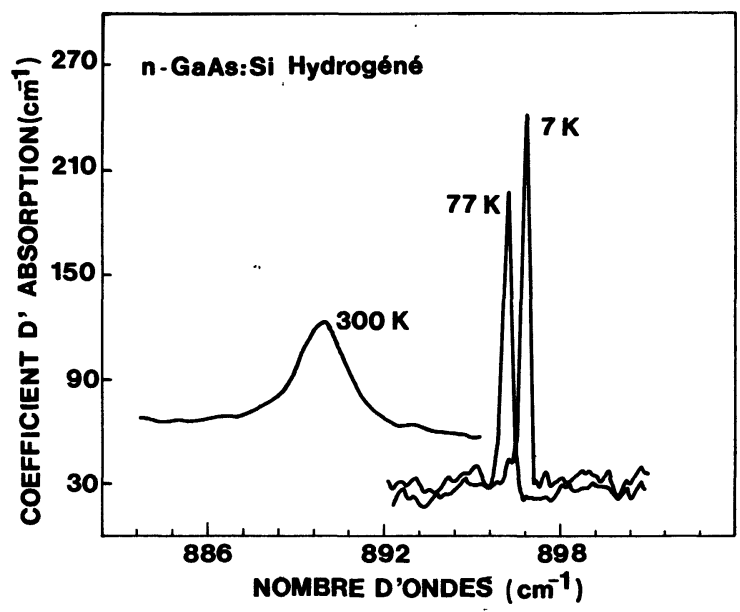

Fig. 4. - Raie d'absorption associée à l'hydrogène dans la région $890 \mathrm{~cm}^{-1}$ dans une couche épitaxiée de $\mathrm{GaAs:Si}(\mathrm{n})$ hydrogénée. La bande à $300 \mathrm{~K}$ a été obtenue pour une couche de $4 \mu \mathrm{m}$ d'épaisseur et un taux de dopage en silicium de $3 \times 10^{18} \mathrm{~cm}^{-3}$. Les bandes à $77 \mathrm{~K}$ et $7 \mathrm{~K}$ correspondent à une couche de $3 \mu \mathrm{m}$ avec [Si] = $1 \times 10^{18} \mathrm{~cm}^{-3}$. La largeur observée à $77 \mathrm{~K}$ et $7 \mathrm{~K}$ est limitée par la résolution de $0,3 \mathrm{~cm}^{-1}$. La largeur réelle de la raie est de $0,08 \mathrm{~cm}^{-1}$ à ces températures.

[Absorption of the hydrogen related band near $890 \mathrm{~cm}^{-1}$ in a hydrogenated n-type silicon doped GaAs epilayer. The band at $300 \mathrm{~K}$ has been obtained for a layer thickness of $4 \mu \mathrm{m}$ and [Si] $=3 \times 10^{18} \mathrm{~cm}^{-3}$. The bands at $77 \mathrm{~K}$ and $7 \mathrm{~K}$ correspond to a sample with a $3 \mu \mathrm{m}$ thick epilayer with [Si] $=1 \times 10^{18} \mathrm{~cm}^{-3}$. The observed width at $77 \mathrm{~K}$ and $7 \mathrm{~K}$ is limited by the resolution of $0.3 \mathrm{~cm}^{-1}$. The actual width at these temperatures is $0.08 \mathrm{~cm}^{-1}$.]

dire voisin de $\sqrt{2}$. Ce résultat prouve directement que l'hydrogène est présent dans le centre à l'origine de la raie à $890 \mathrm{~cm}^{-1}$. On sait aussi que l'hydrogène diffuse dans les cristaux de GaAs non dopés silicium [13]. La non-observation de la raie précédente dans ce type de matériau (Fig. 5a) montre que la liaison de l'hydrogène est associée à la présence d'atomes donneurs silicium dans la matrice.

Il est important désormais de savoir s'il existe un lien entre la présence de cette raie d'absorption et l'effet de neutralisation des donneurs. Dans le but d'éclaircir ce point, nous avons cherché à établir une éventuelle relation entre l'intensité d'absorption de la raie à $890 \mathrm{~cm}^{-1}$ et la concentration de donneurs silicium neutralisés. Pour cette étude, nous avons utilisé deux morceaux différents d'une même couche épitaxiée de $4 \mu \mathrm{m}$ d'épaisseur et dopée à $3 \times 10^{18} \mathrm{~cm}^{-3}$. L'un des morceaux a été placé en configuration R.I.M. pour l'absorption infrarouge et l'autre en configuration de Van der Pauw pour les mesures de transport. Les deux morceaux sont ensuite hydrogénés simultanément à $250^{\circ} \mathrm{C}$ pendant $90 \mathrm{~min}$. Après exposition, la concentration de porteurs est de $1,9 \times 10^{17} \mathrm{~cm}^{-3}$ d'après les mesures de Van der Pauw. L'absorption intégrée de la raie à 


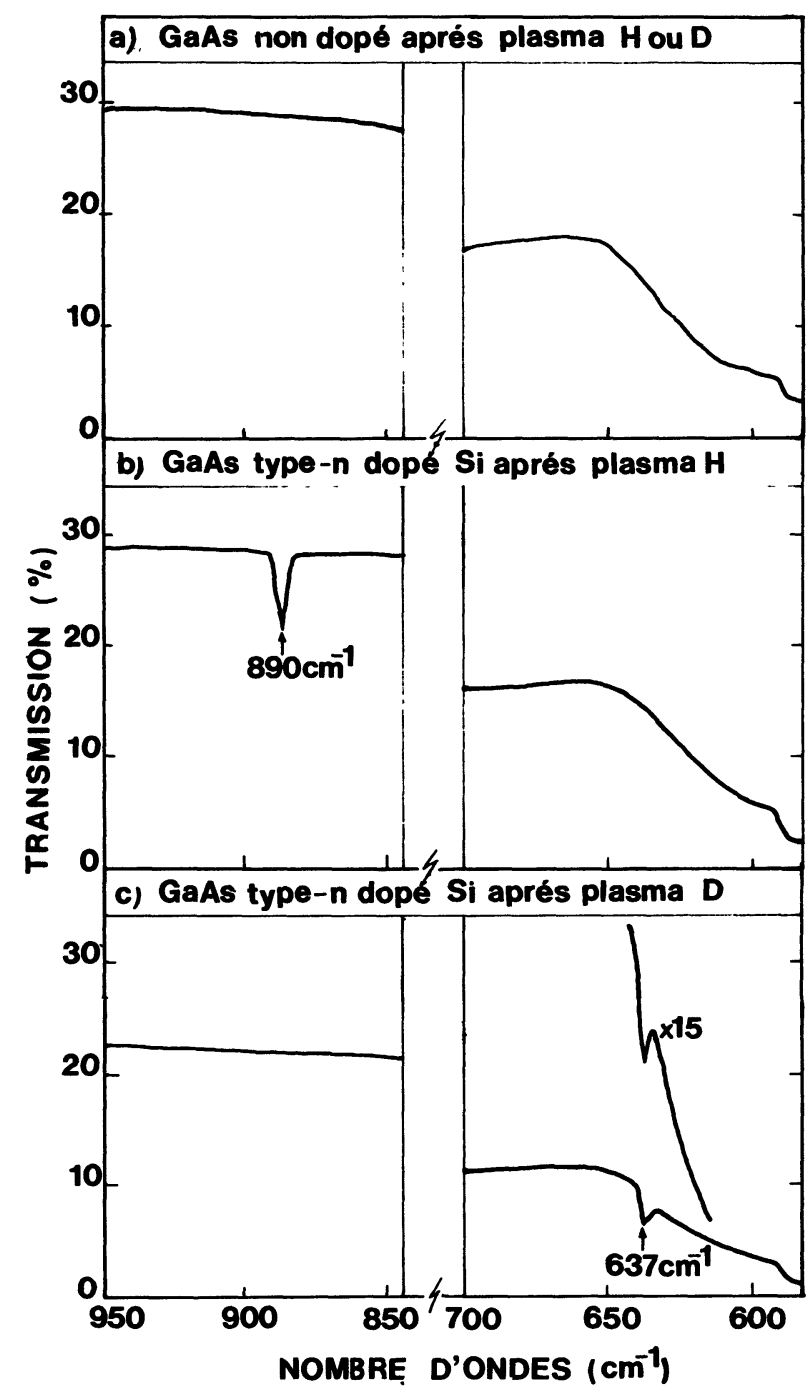

Fig. 5. - Spectre de transmission infrarouge de GaAs hydrogéné et deutéré dans la configuration de réflexion interne multiple. Notons que la bande reliée à l'hydrogène n'apparaît que dans le matériau dopé silicium et est totalement absente dans le matériau non dopé.

[Infrared transmission spectra of hydrogenated and deuterated $\mathrm{GaAs}$ in the multiple internal reflection configuration. Note that the hydrogen related band only appears in the silicon doped sample and is totally absent in the undoped material.]

$890 \mathrm{~cm}^{-1}$ est de $188 \mathrm{~cm}^{-2}$. Les deux échantillons sont ensuite recuits pendant $15 \mathrm{~min}$ à différentes températures comprises entre $250^{\circ} \mathrm{C}$ et $375^{\circ} \mathrm{C}$ dans un mélange azote $(50 \%)$-hydrogène $(50 \%)$. Après chaque recuit, on mesure sur ces mêmes échantillons l'absorption intégrée de la raie à $890 \mathrm{~cm}^{-1}$ et la concentration de porteurs de laquelle on déduit la concentration de donneurs neutralisés. L'ensemble des résultats est reporté sur la figure 6. On observe que l'absorption intégrée de la raie à $890 \mathrm{~cm}^{-1}$ est proportionnelle à la concentration de donneurs silicium neutralisés. L'effet de neutralisation et l'existence du complexe qui donne naissance à la raie

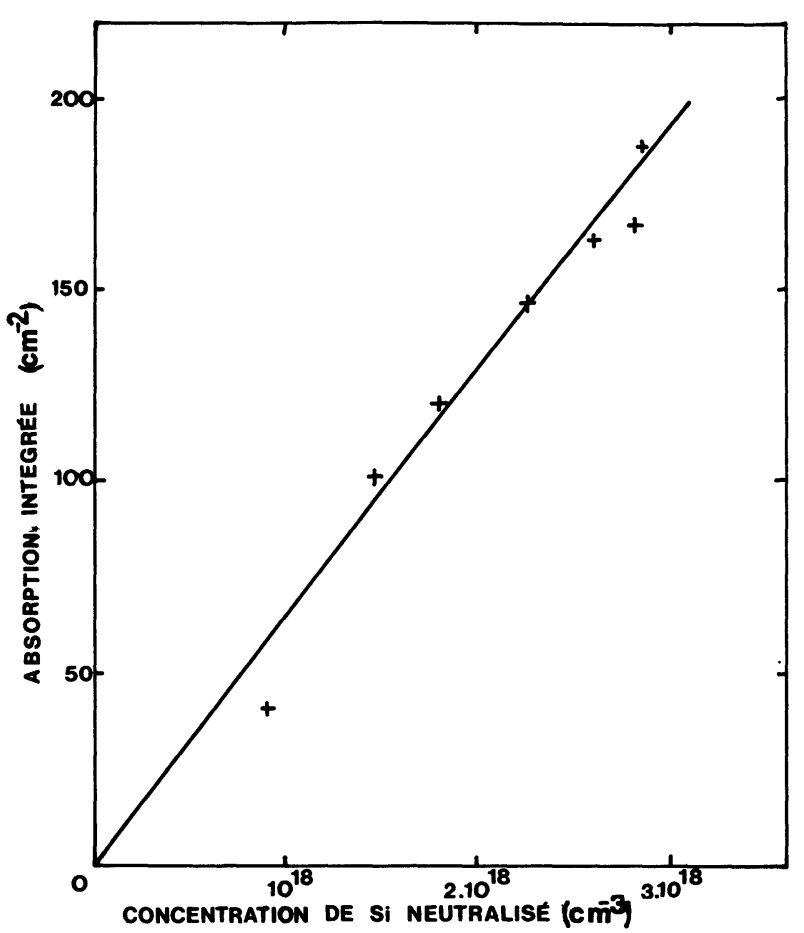

Fig. 6. - Evolution de l'absorption intégrée de la bande à $890 \mathrm{~cm}^{-1}$ avec la concentration de donneurs neutralisés restant après recuit donné d'une couche épitaxiée de GaAs:Si hydrogénée. Les recuits ont été réalisés pendant 15 min à différentes températures comprises entre $250^{\circ} \mathrm{C}$ et $375^{\circ} \mathrm{C}$.

[Integrated absorption of the $890 \mathrm{~cm}^{-1}$ band versus the concentration of neutralized silicon remaining after a given annealing of a hydrogenated silicon doped $\mathrm{GaAs}$ epilayer. The isochronal annealing experiments are performed for $15 \mathrm{~min}$ and extends from $250{ }^{\circ} \mathrm{C}$ to $375^{\circ} \mathrm{C}$.]

d'absorption à $890 \mathrm{~cm}^{-1}$ sont donc étroitement liés ou même ont une origine commune.

\section{Modèle.}

La valeur voisine de $\sqrt{2}$ du rapport des fréquences mesurées dans les matériaux hydrogénés et deutérés montre que la dynamique de l'atome d'hydrogène est une vibration induite par un potentiel vibrationnel classique. Une analyse plus fine de ce rapport est cependant nécessaire si on veut préciser la nature de l'atome auquel l'hydrogène est lié. Les valeurs des fréquences mesurées dans les matériaux hydrogénés et deutérés indiquent, nous l'avons vu plus haut, une valeur de 1,398. Dans le silicium monocristallin, le rapport isotopique associé aux liaisons $\mathrm{Si}-\mathrm{H}$ est de 1,375 [14] alors que dans GaAs monocristallin, il est de 1,390 pour les liaisons $\mathrm{Ga}-\mathrm{H}$ et $\mathrm{Ga}-\mathrm{D}[15,16]$. Cette valeur plus élevée dans le cas de GaAs tient à la masse plus grande du gallium comparée à celle du silicium. La valeur expérimentale de 1,398 suggère donc que l'hydrogène est lié à un atome lourd, c'està-dire soit le gallium, soit l'arsenic. D'autre part, 
dans les conditions d'analyse à haute résolution, les mesures de S.T.F. ont révélé que la raie à $890 \mathrm{~cm}^{-1}$ était unique. L'hydrogène est donc plus probablement lié à l'arsenic qu'au gallium. Dans ce dernier cas, on s'attendrait en effet à ce que la raie à $890 \mathrm{~cm}^{-1}$ soit un doublet associé aux isotopes naturels $\mathrm{Ga}^{69}(60 \%)$ et $\mathrm{Ga}^{71}(40 \%)$. Notre analyse du rapport isotopique des fréquences nous permet de conclure à la formation de liaison arsenic-hydrogène dans $\mathrm{GaAs}$ dopé silicium et hydrogéné. Le dopage par des donneurs est, nous l'avons vu, une condition nécessaire pour l'observation de cette raie. Nous supposons que c'est l'existence de l'électron supplémentaire apporté par le donneur qui est une condition nécessaire pour la formation de la liaison arsenic-hydrogène : cet électron se piégerait pour participer à cette liaison. Pearton et al. ont montré par ailleurs une nette corrélation entre l'énergie d'activation associée à la déneutralisation de différents donneurs et l'énergie de liaison donneurhydrogène [17]. Si on associe à ce résultat l'existence de l'effet de neutralisation, plus précisément la forte diminution après hydrogénation de la concentration de centres ionisés on est amené à penser que l'atome de silicium et la liaison arsenic-hydrogène sont spatialement proches l'un de l'autre. Le modèle le plus simple consiste à supposer que l'arsenic du complexe As-H est en premier voisin d'un atome de silicium. Le complexe serait donc de la forme $\left(\mathrm{SiAs}_{3}\right) \mathrm{As}-\mathrm{H}$.

Des calculs récents du type liaisons fortes ont été réalisés dans le silicium monocristallin dopé bore et hydrogéné. Ils prédisent un état d'énergie minimum pour l'atome d'hydrogène localisé sur le site antiliant d'une liaison bore-silicium [18]. Il serait intéressant d'entreprendre une approche du même type dans GaAs:Si pour se rendre compte de la validité d'un modèle où l'hydrogène serait en site antiliant le long d'une liaison silicium-arsenic.

\section{Stabilité thermique.}

Dans le cadre de ce modèle, un recuit thermique aura pour effet de casser les liaisons arsenic-hydrogène et donc de détruire l'effet de neutralisation. La figure 2 montre la réactivation des donneurs dans une couche épitaxiée, dopée à $1,2 \times 10^{18} \mathrm{~cm}^{-3}$ et hydrogénée. On observe qu'après un recuit à $400{ }^{\circ} \mathrm{C}$ pendant $100 \mathrm{~min}$, la concentration de porteurs et la mobilité des électrons retrouvent presque leur valeur d'origine. L'effet de recuits thermiques successifs sur la déneutralisation des donneurs silicium est illustré sur la figure 7 pour un cristal de GaAs contenant $2,9 \times 10^{18} \mathrm{~cm}^{-3}$ atomes de silicium. Nous avons reporté l'évolution de la fraction de donneurs neutralisés en fonction de la température de recuit. Ce résultat concerne un même cristal recuit, analysé puis recuit, etc... La courbe présente donc le résultat

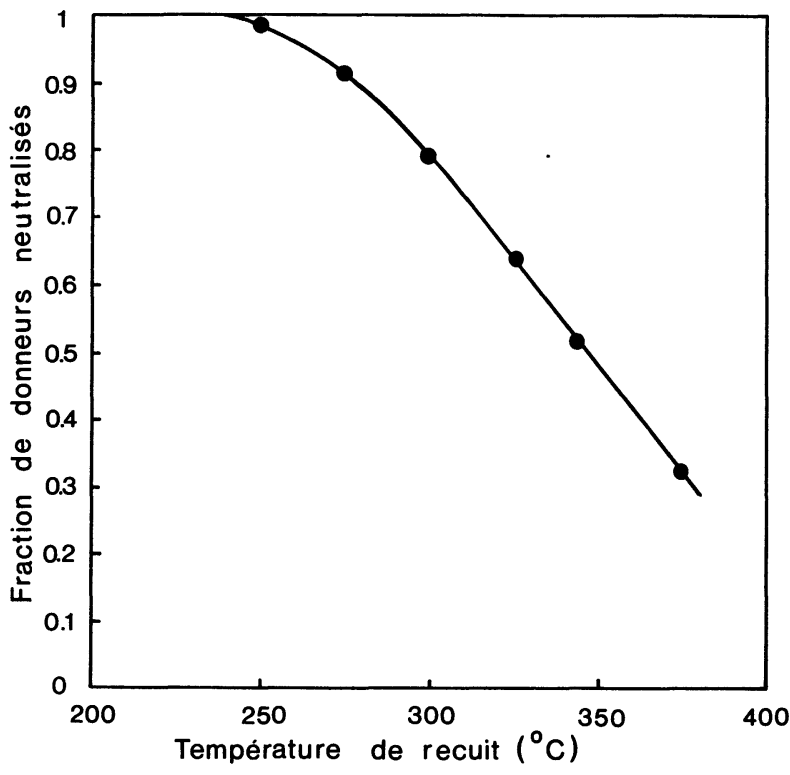

Fig. 7. - Evolution de la fraction de donneurs neutralisés restant après recuit d'une couche épitaxiée de GaAs dopée : $\mathrm{Si}\left([\mathrm{Si}]=2,9 \times 10^{18} \mathrm{~cm}^{-3}\right)$ hydrogénée en fonction de la température de recuit. Chaque recuit a une durée de 15 min. Cette courbe réalisée sur un même cristal reflète le résultat de recuits cumulés.

[Evolution of the fraction of neutralized donors remaining after annealing of a hydrogenated GaAs:Si epilayer $\left([\mathrm{Si}]=2.9 \times 10^{18} \mathrm{~cm}^{-3}\right)$ versus the annealing temperature. The annealing duration is $15 \mathrm{~min}$. This curve obtained using the same epilayer is the result of cumulative annealing effects.]

d'effets cumulés. L'évolution de la fraction de donneurs neutralisés après recuit est sensiblement différente de celle obtenue sur une série d'échantillons de GaAs dopés silicium par implantation sur environ $1000 \AA ̊$ à partir de la surface puis hydrogéné [6]. Il apparaît, en particulier, que l'évolution du rapport $N / N_{0}$ avec la température est moins rapide que celle trouvée dans la référence [6]. Un recuit à $375^{\circ} \mathrm{C}$ pendant $15 \mathrm{~min}$ de $\mathrm{GaAs}$, dopé silicium par implantation puis hydrogéné, réactive quasi complètement les donneurs silicium : il ne reste alors que $1 \%$ de donneurs neutralisés. Dans notre cas, on constate qu'il subsiste encore $32 \%$ de donneurs neutralisếs dans un échantillon ayant subi une série de recuits dont un dernier à $375^{\circ} \mathrm{C}$ pendant $15 \mathrm{~min}$. Suite à ce dernier recuit, la mobilité des électrons dans la couche épitaxiée était de $1935 \mathrm{~cm}^{2} / \mathrm{v} . s$. à $300 \mathrm{~K}$, valeur encore légèrement supérieure à la valeur de $1800 \mathrm{~cm}^{2} / \mathrm{v}$.s. avant hydrogénation.

\section{Conclusions.}

Nous avons montré que l'exposition d'un cristal de GaAs:Si de type $n$ à un plasma d'hydrogène conduit à une diffusion d'atomes d'hydrogène dans le matériau et à un abaissement de la concentration de 
porteurs dans la zone de diffusion de l'hydrogène. $\mathrm{La}$ diminution de la concentration de porteurs s'accompagne d'une augmentation notable de la mobilité électronique. L'analyse de ce phénomène permet de conclure à une diminution de la concentration d'impuretés ionisées, en particulier les donneurs, dans la région hydrogénée. La valeur de la mobilité après hydrogénation est très voisine de celle d'un matériau non hydrogéné de concentration de donneurs actifs équivalente. La réduction de la concentration de porteurs est due, non pas à un effet de compensation, mais à une neutralisation, c'est-àdire à une transformation des donneurs silicium ionisés en complexes neutres. L'étude de spectroscopie infrarouge de GaAs dopé silicium et hydrogéné montre la présence d'une raie d'absorption très fine. La substitution de l'hydrogène par le deutérium entraîne un changement de fréquence dont l'analyse amène à proposer une liaison entre l'arsenic et l'hydrogène. Cette liaison n'existe qu'en présence de donneurs silicium: l'électron supplémentaire du donneur viendrait se repiéger pour participer à la liaison As-H, l'atome de silicium étant supposé en premier voisin de l'arsenic. La formation de ces complexes $\left(\mathrm{SiAs}_{3}\right)$ As-H serait ainsi à la base de l'effet de neutralisation.

\section{Remerciements.}

Nous remercions vivement MM. Martin, Theeten, Guittard et Deconinck du LEP, Limeil-Brévannes pour leurs encouragements constants et leur soutien efficace ainsi que Mlle M. C. Boissy, RTC Compelec, Caen, pour la fourniture de substrats de GaAs:Si. Nous remercions aussi M. Mircea du CNET, Bagneux pour les discussions fructueuses partagées avec lui.

Nous remercions également $M$. Bonnet de la Thomson-CSF, Corbeville, pour les substrats épais de GaAs semi-isolant, M. Laroche et Mme Grattepain du Laboratoire de Physique des Solides de Bellevue pour leur assistance respective dans les mesures d'effet Hall et les mesures à la microsonde ionique.

\section{Bibliographie}

[1] Johnson, N. M., Biegelsen, D. K. et Moyer, M. D., Appl. Phys. Lett. 40 (1982) 882.

[2] Johnson, N. M., Biegelsen, D. K., Moyer, M. D., Deline, V. R. et Evans, C. A. Jr, Appl. Phys. Lett. 38 (1981) 995.

[3] Pankove, J. I., Carlson, D. E., Berkeyheiser, J. E. et Wance, R. O., Phys. Rev. Lett. 51 (1983) 2224.

[4] Pankove, J. I., Zanzucchi, P. J., Magee, C. W. et Lucovsky, G., Appl. Phys. Lett. 46 (1985) 421.

[5] Johnson, N. M., Burnham, R. O., Street, R. A. et Thornton, R. L., Phys. Rev. B 33 (1986) 1102.

[6] Chevallier, J., Dautremont-Smith, W. C., Tu, C. W. et Pearton, S. J., Appl. Phys. Lett. 47 (1985) 108.

[7] Chevallier, J., Dautremont-Smith, W. C., Pearton, S. J., Tu, C. W. et Jalil, A., présenté au $3^{\mathrm{e}}$ Symposium international sur la gravure sèche et le dépôt par plasma en microélectronique, Cachan, France, 26-29 novembre 1985 (édité par Le Vide, les Couches Minces, suppl. $\left.n^{\circ} 229\right) 1985$, p. 161.

[8] Azoulay, R., Dugrand, L., Ankri, D. et Rao, K., J. Cryst. Growth 68 (1984) 453.
[9] Les études de diffusion d'hydrogène ont été réalisées en utilisant un de ses isotopes: le deutérium. Ceci permet d'augmenter notablement la sensibilité dans les mesures à la microsonde ionique.

[10] Chevallier, J., Jalil, A., Azoulay, R. et MirCEA, A., Defects in Semiconductors, édité par H. J. von Bardeleben, Material Science Forum, Vol. 10-12, 591 (1986).

[11] Jalil, A., Chevallier, J., Azoulay, R. et MirCEA, A., J. Appl. Phys. 59 (1986) 3774.

[12] Steeples, K., Dearnaley, G. et Stoneham, A. M., Appl. Phys. Lett. 36 (1980) 981.

[13] Jalil, A., Chevallier, J. (non publié).

[14] BAI, G. R., QI, M. W., XIE, L. M. et SHI, T. S., Solid State Commun. 56 (1985) 277.

[15] Newman, R. C. et Woodhead, J., Radiat. Eff. 53 (1980) 41.

[16] Ascheron, C., Bauer, C., Sobotta, H. et Riede, V., Phys. Status Solidi A 89 (1985) 549.

[17] Pearton, S. J., Dautremont-Smith, W. C., CheVAllier, J., Tu, C. W. et CuMMINGS, K. D., J. Appl. Phys. 59 (1986) 2821.

[18] Johnson, N. M., Herring, C. and Chadi, D. J., Phys. Rev. Lett. 56 (1986) 769. 